\section{Strumpfabgabe in der phlebologischen Praxis}

Seit der Überarbeitung (01.04.2019) steht in der Mittel- und Gegenständeliste (MiGeL) explizit, dass die Abgabestellen für Kompressionsmittel einen Vertrag mit den Krankenversicherern haben müssen. Dies war seit der Einführung des Tarmed und der MiGeL immer schon der Fall. Art. 55 KVV (Verordnung über die Krankenversicherung) regelt unter anderem die Zulassung von Orthopädiefachgeschäften und Apotheken zur Verabreichung von Mitteln und Gegenständen zulasten der OKP (Obligatorischen Krankenpflegeversicherung).
Der jahrzehntealte, unbeanstandete Usus, dass in phlebologisch und/oder gefässmedizinisch spezialisierten Praxen konfektionierte Unterschenkel-Kompressionsstrümpfe angemessen und abgegeben werden können, ist davon nicht betroffen. Die ärztliche Abgabe von Mitteln und Gegenständen fällt unter Art. 35 KVG (Krankenversicherungsgesetz).

Dem SGP-Vorstand sind einige Fälle bekannt geworden, wo niedergelassene Phlebologinnen und Phlebologen neuerdings mit Beanstandungen ihrer Strumpfabgabe konfrontiert wurden. Die SGP (Schweizerische Gesellschaft für Phlebologie) ist dabei, dieses Problem in Kooperation mit der USGG (Union der Schweizerischen Gesellschaften für Gefäßkrankheiten) zu klären. 